\title{
A122 TWIST1 AND HOPX DETERMINE FUNCTION AND PERSISTENCE OF PATHOGENIC MEMORY/EFFECTOR T CELLS
}

Inka Albrecht, Kristyna Hradilkova, Kerstin Westendorf, Melanie Weber, Andreas Radbruch, Hyun-Dong Chang Cell Biology Group, Deutsches RheumaForschungszentrum Berlin, a Leibniz Institute, Berlin, Germany

\subsection{6/ard.2010.148981.25}

Introduction $\mathrm{T}$ helper (Th) lymphocytes support the initiation and maintenance of autoimmune inflammation through secretion of cytokines and provision of B cell help leading to the generation of longlived plasma cells secreting pathogenic autoantibodies. In particular Th type 1 (Th1) and Th17 cells, which promote inflammation through the secretion of IFN- $\gamma$, tumour necrosis factor- $\alpha$ and interleukin-17, have been implicated in the pathogenesis of autoimmunity. The critical role of $\mathrm{CD}^{+}$Th cells has been demonstrated by the clinical benefit achieved through the depletion of Th cells with an anti-CD4 antibody in refractory rheumatoid arthritis patients. However, unwanted side-effects of sustained CD4 lymphopenia and the associated induced immunodeficiency call for the development of more advanced approaches allowing the selective elimination of disease-causing Th cells. This requires a better understanding of the biology of pathogenic Th cells and the identification of selective biomarkers.

Material and methods The authors wanted to investigate the mechanisms involved in the transition from acute to chronic inflammation and identify selective biomarkers allowing the identification of pathogenic Th cells. Following the rationale that Th cells involved in chronic autoimmune inflammation have a history of repeated (auto-)antigen contact, the authors have compared the transcriptomes of Th1 and Th2 cells which they have stimulated only once to Th cells which they have stimulated several times.

Results The authors could identify the two genes, twist1 and hopx, to be specifically upregulated in repeatedly stimulated, pro-inflammatory Th1 cells. Twist1 is a helix-loop-helix transcription factor whose expression increments with each restimulation. Twist 1 is highly expressed in $\mathrm{CD}^{+} \mathrm{T}$ cells isolated directly from the site of inflammation in patients with, for example, rheumatoid arthritis and inflammatory bowel disease, but not in T cells isolated from the peripheral blood. When twist1 is downregulated in Th1 cells by RNA interference in the murine ovalbumin-induced arthritis model, inflammation is augmented, indicating that twist 1 is an intrinsic regulator of inflammation. Inhibition of hopx expression in Th1 cells leads to an impairment in their ability to persist in vivo. Accordingly, hopx-deficient mice have fewer effector/memory Th1 cells in steady-state. Th1 cells, which lack 
expression of hopx, are not able to induce inflammation in the murine transfer colitis model or the ovalbumin-induced arthritis model.

Conclusions Twist1 and hopx qualify as selective biomarkers allowing the identification of pro-inflammatory, pathogenic Th1 cells. The importance of twist1 and hopx for the function and persistence of pathogenic Th1 cells may offer novel opportunities for the selective targeting of such cells. 